\title{
Moderation Analysis
}

Contents

8.1 Introduction - 156

8.2 Types of Moderator

Variables - 157

8.3 Modeling Moderating Effects - 158

8.4 Creating the Interaction

Term - 159

8.5 Model Evaluation - 161

8.6 Result Interpretation - 162

8.7 Case Study Illustration: Moderation Analysis - 164

References - 171 


\section{Learning Objectives}

After reading this chapter, you should:

1. Comprehend the basic concepts of moderation when using PLS-SEM

2. Be able to technically execute a moderation analysis

3. Understand how to interpret the results of a moderation analysis

4. Learn how to execute a slope analysis

5. Be able to use SEMinR to conduct a moderation analysis based on the corporate reputation example

\subsection{Introduction}

Moderation describes a situation in which the relationship between two constructs is not constant but depends on the values of a third variable, referred to as a moderator variable. The moderator variable (or construct) changes the strength, or even the direction of a relationship between two constructs in a model. For example, prior research has shown that the relationship between customer satisfaction and customer loyalty differs as a function of the customers' income or age (e.g., Homburg \& Giering, 2001). More precisely, income has a pronounced negative effect on the satisfaction to loyalty relationship - the higher the income, the weaker the relationship between satisfaction and loyalty. In short, income serves as a moderator variable that accounts for heterogeneity in the data. This means the satisfaction to loyalty relationship is not the same for all customers but differs depending on the income level. In this respect, moderation can (and should) be seen as a means to account for heterogeneity in the data.

Moderating relationships are hypothesized a priori by the researcher. The testing of the moderating relationship depends on whether the researcher hypothesizes whether one specific model relationship or whether all model relationships depend on the values of the moderator. Moderators can be either single items or multiitem constructs. In our satisfaction-loyalty example, we hypothesized that only the satisfaction to loyalty relationship is influenced by income. These considerations also apply for the corporate reputation model and its relationship between CUSA and CUSL. In such a setting, we would, for example, examine if and how the respondents' income influences the relationship between CUSA and CUSL. - Figure 8.1 shows the theoretical model of such a moderating relationship.

Alternatively, we could also hypothesize that several relationships in the corporate reputation model depend on some customer characteristic, such as gender. In this case, we would run a multigroup analysis (Klesel, Schuberth, Niehaves, \& Henseler, 2021). For a detailed explanation of multigroup analysis in PLS-SEM, see Chap. 8 in Hair et al. (2022) and Chap. 4 in Hair, Sarstedt, Ringle, and Gudergan (2018). In this chapter, our focus is on the (single) moderator analysis. More specifically, we address the modeling and interpretation of an interaction effect that occurs when a moderator variable is assumed to influence one specific relationship. 
- Fig. 8.1 Theoretical moderation model example. (Source: Hair, Hult, Ringle, \& Sarstedt, 2022, Chap. 7; used with permission by Sage)

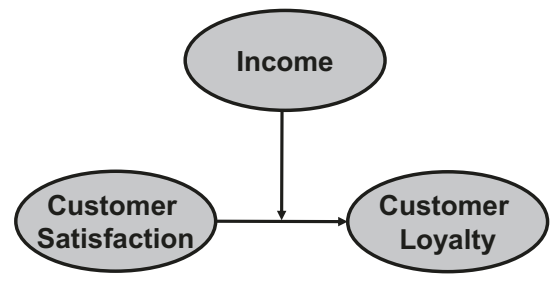

\subsection{Types of Moderator Variables}

Moderators can be present in structural models in different forms. They can represent observable traits, such as gender, age, or income. But they can also represent unobservable traits, such as risk attitude, attitude toward a brand, or ad liking. Moderators can be measured with a single item or multiple items and using reflective or formative indicators. The most important differentiation, however, relates to the moderator's measurement scale, which involves distinguishing between categorical (typically dichotomous) and continuous moderators.

In our corporate reputation case study in the mobile phone industry, we could, for example, use the service-type variable (contract versus prepaid) as a categorical moderator variable. These categorical variables are usually dummy coded (i.e., 0/1), whereby the zero (" 0 ") represents one of the two categories, called the reference category, while the value one ("1") represents the other category in a two-category situation. Note, however, that a categorical moderator can represent more than two groups. For example, in the case of three groups (e.g., short-term contract, long-term contract, and prepaid), we could divide the moderator into two dummy variables, which are simultaneously included in the model. In the latter case, both dummy variables would take the value zero for the reference category (e.g., prepaid). The other two categories would be indicated by the value 1 in the corresponding dummy variable.

Similar to regression analysis, categorical moderators can be included in a PLS path model when specifying the structural model. For example, in the case study on corporate reputation, we could evaluate whether the customers' gender has a significant bearing on the satisfaction-loyalty link. In many cases, however, researchers use a categorical moderator variable to split up the dataset into two or more groups and then estimate the models separately for each group of data. Running a multigroup analysis enables identification of model relationships that differ significantly between the groups (Hair et al., 2018, Chap. 4). This approach offers a more complete picture of the moderator's influence on the analysis results as the focus shifts from examining its impact on one specific model relationship to examining its impact on all model relationships.

In many situations, researchers have a continuous moderator variable that they theorize will affect the strength of one specific relationship between two latent variables. Returning to our case study on corporate reputation, we could, for example, hypothesize that the relationship between satisfaction and loyalty is influenced by the customers' income. More precisely, we could hypothesize that the relationship between customer satisfaction and customer loyalty is weaker for high-income cus- 
tomers and stronger for low-income customers. This type of moderator effect would indicate the satisfaction to loyalty relationship changes depending on the level of income. If this income moderator effect is not present, we would assume the strength of the relationship between satisfaction and loyalty is constant.

Continuous moderators are typically measured with multi-item constructs but can, in principle, also be measured using only a single item. When the moderator variable represents some abstract unobservable trait (as opposed to some observable phenomenon, such as income), however, we clearly advise against the use of single items for construct measurement. In short, multi-item scales are much more effective in terms of explaining the target construct's variance (Diamantopoulos, Sarstedt, Fuchs, Kaiser, \& Wilczynski, 2012; Sarstedt, Diamantopoulos, Salzberger, \& Baumgartner, 2016), which can be particularly problematic in the context of moderation. The reason is that moderation is usually associated with rather limited effect sizes (Aguinis, Beaty, Boik, \& Pierce, 2005), so small (but meaningful) effects will be more difficult to identify as significant. Furthermore, when modeling moderating effects, the moderator's measurement model is included twice in the model - in the moderator variable itself and in the interaction term (see the next section). This characteristic amplifies the limitations of single-item measurement(s) in research situations involving moderation.

\subsection{Modeling Moderating Effects}

To gain an understanding of how moderating effects are modeled, consider the path model shown in - Fig. 8.2. This model illustrates our previous example in which income serves as a moderator variable $(M)$, influencing the relationship between customer satisfaction $\left(Y_{1}\right)$ and customer loyalty $\left(Y_{2}\right)$. The moderating effect $\left(p_{3}\right)$ is represented by an arrow pointing at the effect $p_{1}$ linking $Y_{1}$ and $Y_{2}$. Furthermore, when including the moderating effect in a PLS path model, there is also a direct relationship $\left(p_{2}\right)$ from the moderator to the endogenous construct. This additional path is important (and a frequent source of mistakes), as it controls for the direct impact of the moderator on the endogenous construct. If the path $p_{2}$ was to be omitted, the effect of $M$ on the relationship between $Y_{1}$ and $Y_{2}$ (i.e., $p_{3}$ ) would be inflated. As can be seen, moderation is somewhat similar to mediation, in that a third variable (i.e., a mediator or moderator variable) affects the strength of a relationship between two latent variables. The crucial distinction between both concepts is that the moderator variable does not depend on the exogenous construct. In contrast, with mediation, there is a direct effect between the exogenous construct and the mediator construct (Memon et al., 2018).

- Fig. 8.2 Moderation model example. (Source: Hair et al., 2022, Chap. 7; used with permission by Sage)

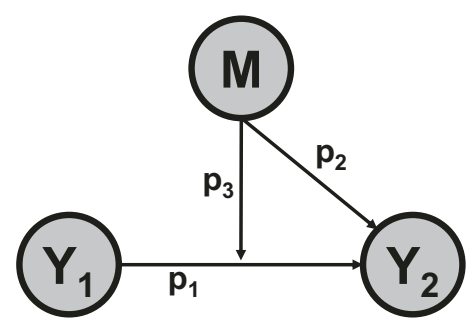


The path model in - Fig. 8.2, which includes a moderating effect, can also be expressed mathematically using the following formula:

$$
Y_{2}=\left(p_{1}+p_{3} \cdot M\right) \cdot Y_{1}+p_{2} \cdot M
$$

As can be seen, the influence of $Y_{1}$ on $Y_{2}$ not only depends on the strength of the simple effect $p_{1}$ but also on the product of $p_{3}$ and $M$. To understand how a moderator variable can be integrated in a path model, we need to rewrite the equation as follows:

$$
Y_{2}=p_{1} \cdot Y_{1}+p_{2} \cdot M+p_{3} \cdot\left(Y_{1} \cdot M\right) .
$$

This equation shows that including a moderator effect requires the specification of the effect of the exogenous construct (i.e., $p_{1} \cdot Y_{1}$ ), the effect of the moderator variable (i.e., $\left.p_{2} \cdot M\right)$, and the product term $p_{3} \cdot\left(Y_{1} \cdot M\right)$, which is also called the interaction term. As a result, the coefficient $p_{3}$ expresses how the effect $p_{1}$ changes when the moderator variable $M$ is increased or decreased by one standard deviation unit. $\bullet$ Figure 8.3 illustrates the concept of an interaction term. As can be seen, the model includes the interaction term as an additional latent variable covering the product of the exogenous construct $Y_{1}$ and the moderator $M$. Because of this interaction term, researchers often refer to interaction effects when modeling moderator variables.

So far, we have looked at a two-way interaction because the moderator interacts with one other variable, the exogenous construct $Y_{1}$. However, it is also possible to analyze a multiple moderator model. In such a model, the researcher can include a twoway interaction term for each moderator and the moderated relationship into the same model (e.g., when income and age both affect the customer to loyalty relationship in a moderation analysis). When using multiple moderators, it is also possible to model higher levels of interaction (e.g., a three-way interaction term), where the moderating effect (itself) is again moderated. Such a setup is also referred to as cascaded moderator analysis. The most common form of a cascaded moderator analysis is a three-way interaction (Henseler \& Fassott, 2010). For example, we could imagine that the moderating effect of income is not constant but is itself influenced by some other variable, such as age, which would then serve as a second moderator variable in the model.

\subsection{Creating the Interaction Term}

In the previous section, we introduced the concept of an interaction term to facilitate the inclusion of a moderator variable in a PLS path model. But a fundamental question remains: How should the interaction term be operationalized? Research

- Fig. 8.3 Interaction term in moderation. (Source: Hair et al., 2022, Chap. 7; used with permission by Sage)

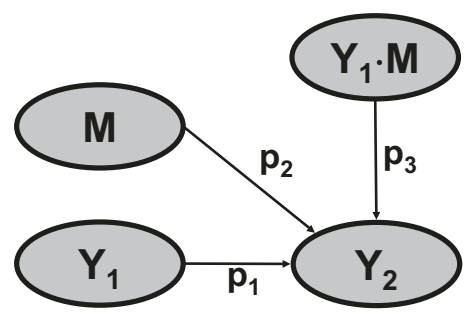


has proposed three primary approaches for creating the interaction term: (1) the product indicator approach, (2) the orthogonalizing approach, and (3) the twostage approach. Simulation studies have shown that Chin, Marcolin, and Newsted's (2003) two-stage approach excels in terms of parameter recovery and statistical power (e.g., Becker, Ringle, \& Sarstedt, 2018; Henseler \& Chin, 2010). In addition, this approach offers much flexibility, as it is the only approach that is applicable when the exogenous construct ( $Y_{1}$ in $\mathbf{D}$ Fig. 8.3) or the moderator ( $M$ in $\square$ Fig. 8.3) is specified formatively. We therefore recommend using the two-stage approach in most situations to create the interaction term. In the following, we discuss the twostage approach in greater detail. See Chap. 7 in Hair et al. (2022) for a discussion of the product indicator approach and the orthogonalizing approach.

The two-stage approach has its roots in its explicit exploitation of PLS-SEM's advantage to estimate latent variable scores (Becker et al., 2018; Rigdon, Ringle, \& Sarstedt, 2010). The two stages are as follows:

- Stage 1: the main effect model (i.e., without the interaction term) is estimated to obtain the scores of the latent variables. These are saved for further analysis in Stage 2.

- Stage 2: the latent variable scores of the exogenous construct and moderator variable from Stage 1 are multiplied to create a single item used to measure the interaction term. All other latent variables are represented by means of single items of their latent variable scores from Stage 1.

- Figure 8.4 illustrates the two-stage approach for our previous model, where two formative indicators are used in Stage 1 to measure the moderator variable. The main effect model in Stage 1 is run to obtain the latent variable scores for $Y_{1}, Y_{2}$, and $M$ (i.e., $\operatorname{LVS}\left(Y_{1}\right), \operatorname{LVS}\left(Y_{2}\right)$, and $\operatorname{LVS}(M)$ ). The latent variable scores of $Y_{1}$ and $M$ are then multiplied to form the single item used to measure the interaction term $Y_{1} \cdot M$ in Stage 2. The latent variables $Y_{1}, Y_{2}$, and $M$ are each measured with a single item of the latent variable scores from Stage 1. It is important to note that the limitations identified when using single items do not apply in this case, since the single item represents the latent variable scores as obtained from a multi-item measurement in Stage 1.

- Fig. 8.4 Two-stage approach. (Source: Hair et al., 2022, Chap. 7; used with permission by Sage)

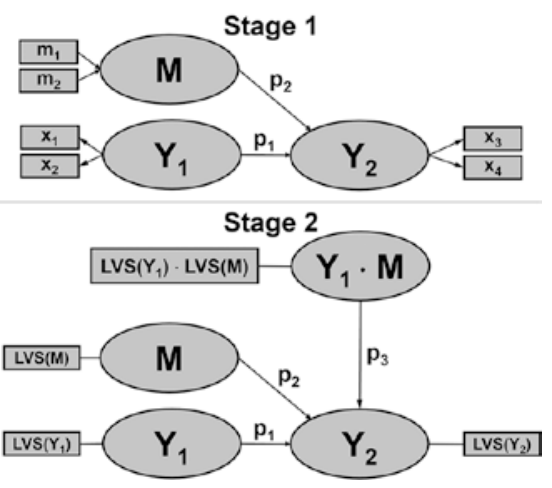


Becker et al. (2018) examined the impact of different data treatment options on the two-stage approach's performance. The results show parameter recovery works best when standardizing the indicator data and the interaction term rather than working with unstandardized or mean-centered data. Standardization is done by subtracting the variable's mean from each observation and dividing the result by the variable's standard error (Sarstedt \& Mooi, 2019; Chap. 5). As indicated previously and in consideration of the above advantages, we recommend that in most situations, researchers apply the two-stage approach with standardized data when conducting moderator analyses.

\subsection{Model Evaluation}

Measurement and structural model evaluation criteria, as discussed in $\boldsymbol{D}$ Chaps. 4,5 , and 6, also apply to moderator models. For the interaction term, however, there is no requirement to assess its measurement model since it represents an auxiliary measurement that incorporates the interrelationships between the moderator and the exogenous construct in the path model. This characteristic, however, renders any measurement model assessment of the interaction term meaningless. In addition, standard measurement model evaluation standards would not apply since the interaction term is measured with a single item. Therefore, the interaction term does not necessarily have to be assessed in the measurement model evaluation step.

\section{Important}

There is no requirement to assess the measurement model of the interaction term since it represents an auxiliary measurement that does not represent a distinct theoretical entity.

Finally, it is always important to consider the standard criteria for structural model assessment. In the context of moderation, particular attention should be paid to the $f^{2}$ effect size of the interaction effect (Hair et al., 2022; Memon et al., 2018). As explained in Chap. 6, this criterion enables an assessment of the change in the $\mathrm{R}^{2}$ value when an exogenous construct is omitted from the model. With regard to the interaction effect, the $f^{2}$ effect size indicates how much the moderation contributes to the explanation of the endogenous construct. The effect size can be calculated as

$$
f^{2}=\frac{R_{\text {included }}^{2}-R_{\text {excluded }}^{2}}{1-R_{\text {included }}^{2}},
$$

where $\mathrm{R}_{\text {included }}^{2}$ and $\mathrm{R}_{\text {excluded }}^{2}$ are the $\mathrm{R}^{2}$ values of the endogenous construct when the interaction term of the moderator model is included in or excluded from the PLS path model. In this way, one can assess the relevance of the moderating effect. General guidelines for assessing $f^{2}$ suggest values of $0.02,0.15$, and 0.35 represent 
small, medium, and large effect sizes, respectively (Cohen, 1988). However, Aguinis et al. (2005) have shown that the average effect size in tests of moderation is only 0.009 . Against this background, Kenny (2018) proposes that $0.005,0.01$, and 0.025 , respectively, constitute more realistic standards for small, medium, and large effect sizes of moderation but also points out that even these values are optimistic.

\section{( Important}

Standard cutoff values for the $f^{2}$ effect size do not apply when interpreting the interaction term's impact. Instead $f^{2}$ values of $0.005,0.01$, and 0.025 should be considered as evidence for small, medium, and large effect sizes, respectively.

\subsection{Result Interpretation}

When interpreting the results of a moderation analysis, the primary focus is the significance of the interaction term. If the interaction term's effect on the endogenous construct is significant, we conclude the moderator $M$ has a significant moderating effect on the relationship between $Y_{1}$ and $Y_{2}$. The bootstrapping procedure ( Chap. 5) facilitates this assessment. If the relationship is statistically significant, the next step is to determine the strength of the moderating effect.

In a model without moderation (i.e., without the moderator variable $M$ ) in which there is only an arrow linking $Y_{1}$ and $Y_{2}$ (see - Fig. 8.3), the effect $p_{1}$ is referred to as a direct effect or main effect. In the case of the two-stage approach, such a main effect is, however, different from the corresponding relationship in a moderator model shown in - Fig. 8.3. Here, in contrast, $p_{1}$ is referred to as a simple effect, expressing the effect of $Y_{1}$ on $Y_{2}$ that is moderated by $M$. More specifically, the estimated value of $p_{1}$ represents the strength of the relationship between $Y_{1}$ and $Y_{2}$ when the moderator variable $M$ has a value of zero. If the level of the moderator variable is increased (or decreased) by one standard deviation unit, the simple effect $p_{1}$ is expected to change by the size of $p_{3}$. For example, if the simple effect $p_{1}$ equals 0.30 and the moderating effect $p_{3}$ has a value of -0.10 , one would expect the relationship between $Y_{1}$ and $Y_{2}$ to decrease to a value of $0.30+(-0.10)=0.20$, if (ceteris paribus) the mean value of the moderator variable $M$ increases by one standard deviation unit (Henseler \& Fassott, 2010). As a result, a moderator variable can strengthen, weaken, or even reverse a relationship (Gardner, Harris, Li, Kirkman, \& Mathieu, 2017).

In many model setups, however, zero is not a number on the scale of $M$ or, as in the case in our example (i.e., it is not a meaningful value for the moderator). If this is the case, the interpretation of the simple effect becomes problematic. This is another reason why we need to standardize the indicators of the moderator as described earlier. The standardization shifts the reference point from an income of zero to the average income and thus facilitates interpretation of the effects.

An important concept to understand is that the nature of the effect between $Y_{1}$ and $Y_{2}$ (i.e., $p_{1}$ ) differs for models with and without the moderator when using the two-stage approach. If the focus is on testing the significance of the main effect $p_{1}$ 
between $Y_{1}$ and $Y_{2}$, the PLS-SEM analysis should be initially executed without the moderator. The evaluation and interpretation of results should follow the procedures outlined in Chap. 6 . The moderator analysis then is executed as a complementary analysis for the specific moderating relationship. This issue is important because the direct effect becomes a simple effect in the moderator model, which differs in its estimated value, meaning, and interpretation. The simple effect represents the relationship between an exogenous and an endogenous construct when the moderator variable's value is equal to its mean value (provided standardization has been applied). Hence, interpreting the simple effect results of a moderator model as if it were a direct effect (e.g., for testing the hypothesis of a significant relationship $p_{1}$ between $Y_{1}$ and $Y_{2}$ ) may result in misleading and incorrect conclusions (Henseler \& Fassott, 2010).

8 When testing a hypothesized direct relationship between two constructs, the moderator needs to be excluded from the model.

Beyond understanding these aspects of moderator analysis, the interpretation of moderation results is often quite challenging. For this reason, graphical illustrations of results support their understanding and drawing of conclusions. A common way to illustrate the results of a moderation analysis is by slope plots (Memon et al., 2018).

\section{Tip}

Web pages, such as those by Jeremy Dawson ( http://www.jeremydawson.co.uk/ slopes.htm) or Kristopher Preacher ( http://quantpsy.org/interact/mlr2.htm), provide online tools for corresponding computations and simple slope plot extractions.

As an example of a two-way interaction, refer to 0 Fig. 8.3. Assume the relationship between $Y_{1}$ and $Y_{2}$ has a value of 0.50 , the relationship between $M$ and $Y_{2}$ has a value of 0.10 , and the interaction term $\left(Y_{1} \cdot M\right)$ has a 0.25 relationship with $Y_{2}$. - Figure 8.5 shows a typical slope plot used for such a setting, where the $x$-axis represents the exogenous construct $\left(Y_{1}\right)$ and the $y$-axis the endogenous construct $\left(Y_{2}\right)$.

The two lines in 0 Fig. 8.5 represent the relationship between $Y_{1}$ and $Y_{2}$ for low and high levels of the moderator construct $M$. Usually, a low level of $M$ is one standard deviation unit below its average (straight line in - Fig. 8.5), while a high level of $M$ is one standard deviation unit above its average (dotted line in $\boldsymbol{0}$ Fig. 8.5). Because of the positive moderating effect, as expressed in the 0.25 relationship between the interaction term and the endogenous construct, the high moderator line's slope is steeper. That is, the relationship between $Y_{1}$ and $Y_{2}$ becomes stronger with high(er) levels of $M$. For low(er) levels of $M$, the slope is much flatter, as shown in - Fig. 8.5. Hence, with low(er) levels of the moderator construct $M$, the relationship between $Y_{1}$ and $Y_{2}$ becomes weaker. 


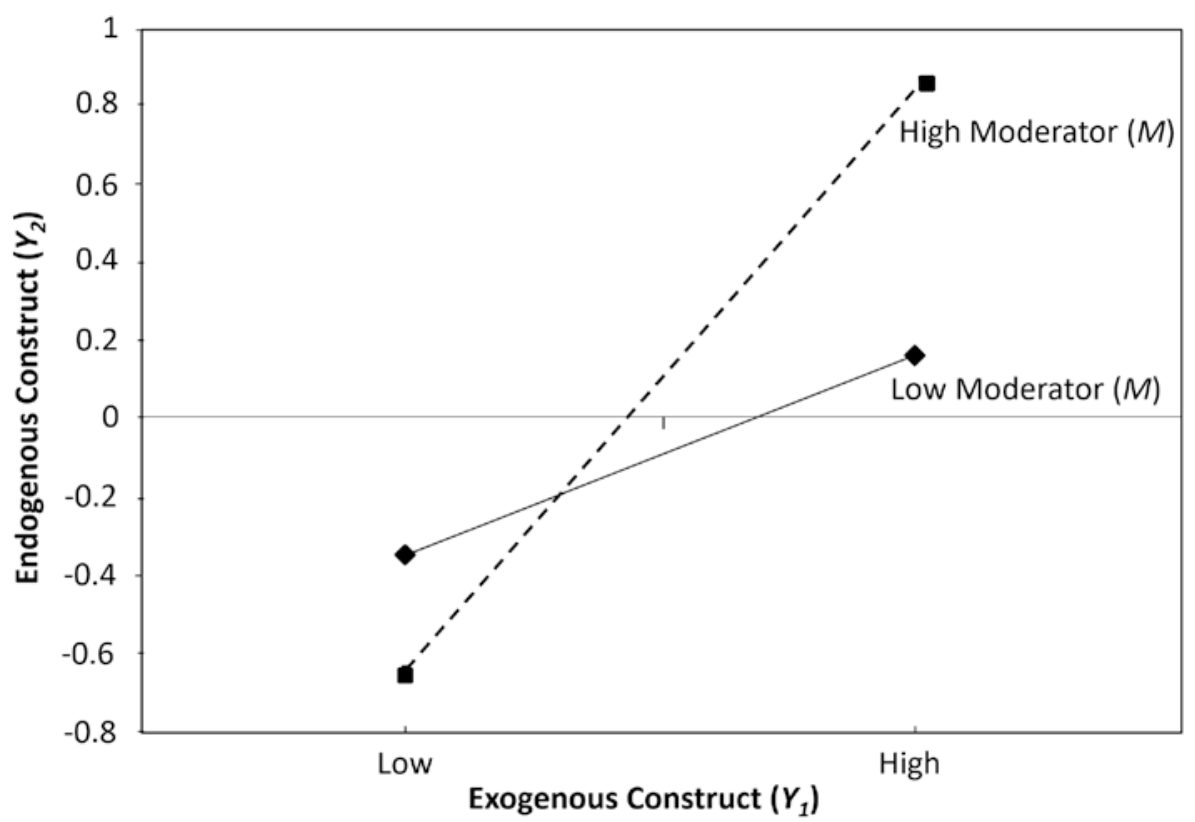

- Fig. 8.5 Slope plot. (Source: Hair et al., 2022, Chap. 7; used with permission by Sage)

\subsection{Case Study Illustration: Moderation Analysis}

We again turn to the corporate reputation model as discussed in the previous chapters to illustrate the moderation concepts. In the subsequent discussion, we focus on the relationship between customer satisfaction and customer loyalty. Specifically, we introduce perceived switching costs as a moderator variable that can be assumed to negatively influence the relationship between satisfaction and loyalty (Hair et al., 2022; Chap. 7). The higher the perceived switching costs, the weaker the relationship between these two constructs (- Fig. 8.6). We use an extended form of Jones, Mothersbaugh, and Beatty's (2000) scale and measure switching costs reflectively using four indicators (switch_l to switch_4; - Table 8.1), each measured on a 5 -point Likert scale ( 1 = fully disagree, $5=$ fully agree $)$.

First, we need to update the measurement and structural models to include the new $S C$ construct and its indicators switch_1, switch_2, switch_3, and switch_4. We thus need to add a new element to the list of constructs in the measurement model using the composite () function. We name the construct "SC" and specify the items using the multi_items() function and arguments "switch_" and "1:4": composite("SC", multi_items("switch_", 1:4)).

Creating interaction terms by hand can be time-consuming and error prone. SEMinR provides functions for simply creating interactions between constructs. In doing so, SEMinR adjusts the standard errors of the construct scores in the generation of the interaction term (Henseler \& Chin, 2010). Interaction 
Table 8.1 Switching costs indicators

\begin{tabular}{|l|l|}
\hline $\begin{array}{l}\text { Construct } \\
\text { name }\end{array}$ & Construct definition \\
\hline switch_1 & It takes me a great deal of time to switch to another company \\
\hline switch_2 & It costs me too much to switch to another company \\
\hline switch_3 & $\begin{array}{l}\text { It takes a lot of effort to get used to a new company with its specific "rules" } \\
\text { and practices }\end{array}$ \\
\hline switch_4 & \begin{tabular}{l} 
In general, it would be a hassle switching to another company \\
\hline Source: authors' own table; Jones et al. (2000)
\end{tabular} \\
\hline
\end{tabular}

- Fig. 8.6 Corporate reputation model with the added moderator switching costs $(S C)$ and the interaction term (CUSA

* $S C$ ). (Source: authors' own figure)

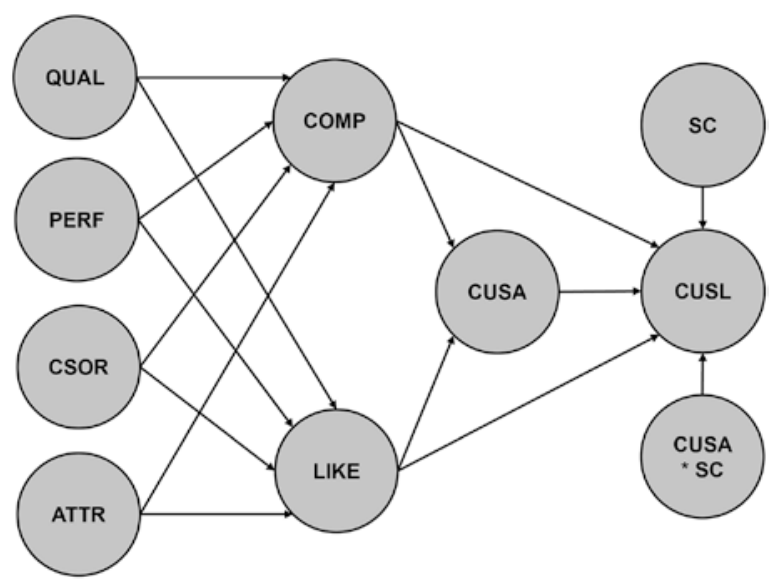

terms are described in the measurement model function constructs () using the following methods:

- two stage implements the two-stage approach as recommended in our previous discussions. It specifies the interaction term as the product of the scores of the exogenous construct and the moderator variable.

- product_indicator generates the interaction term by multiplying each indicator of the exogenous construct with each indicator of the moderator variable.

- orthogonal is an extension of the product indicator approach, which generates an interaction term whose indicators do not share any variance with the indicators of the exogenous construct and the moderator. The orthogonalizing approach is typically used to handle multicollinearity in the structural model.

The SEMinR syntax uses an asterisk ("*”) as a naming convention for the interaction construct. Thus, when creating an interaction from the constructs $C U S A$ and $S C$, the resulting interaction is called "CUSA*SC" in the structural model. We therefore recommend refraining from using an asterisk in the naming of noninteraction constructs. 
In addition to including the $S C$ construct, we need to specify the interaction term composed of the independent variable CUSA and the moderator $S C$ using the interaction_term () function. The interaction_term () function takes the following arguments: $i v$ specifies the independent variable (i.e., exogenous construct); moderator specifies the moderating variable; and method specifies the interaction calculation method (with values product_indicator, orthogonal, or two_stage). We now need to choose the two-stage approach to create the interaction term. We specify the interaction term as follows: interaction term $(i v=$ "CUSA", moderator $=$ "SC", method $=$ two_stage $)$. SEMinR automatically generates a name for the new interaction term by combining the exogenous construct's and the moderator construct's name separated by an asterisk (“*”), representing multiplication. Thus, the newly created interaction term will be called $C U S A * S C$. Note that SEMinR always standardizes the data when calculating the interaction term.

\# Load the SEMinR library

library (seminr)

\# Load the data

corp_rep_data <- corp_rep_data

\# Create the measurement model

corp_rep_mm_mod <- constructs (

composite ("QUAL", multi_items ("qual_", 1:8), weights = mode_B), composite("PERF", multi_items ("perf_", 1:5), weights = mode_B), composite ("CSOR", multi_items ("CSOr_", 1:5), weights = mode_B), composite("ATTR", multi_items ("attr_", 1:3), weights = mode_B), composite("COMP", multi_items ("comp_", 1:3)), composite("LIKE", multi_items ("like_", 1:3)), composite("CUSA", singlēeitem("Cusa")), composite("SC", multi_items("switch_", 1:4)), composite ("CUSL", multi_items ("cusl_", 1:3)), interaction_term( $i_{V}=$ "C $\mathrm{USA}$ ", moderator $=$ "SC", method = two stage))

When the measurement model has been updated, we need to add the new structural paths. There are two new paths in the model: from $S C$ to $C U S L$ and from the interaction term $C U S A^{*} S C$ to $C U S L$. We therefore append the list with the two new construct names: paths (from $=c$ ("CUSA", "SC", "CUSA*SC"), to $=c($ "CUSL") $)$. With the measurement and structural models now updated, the model can be estimated, bootstrapped, and summarized. 


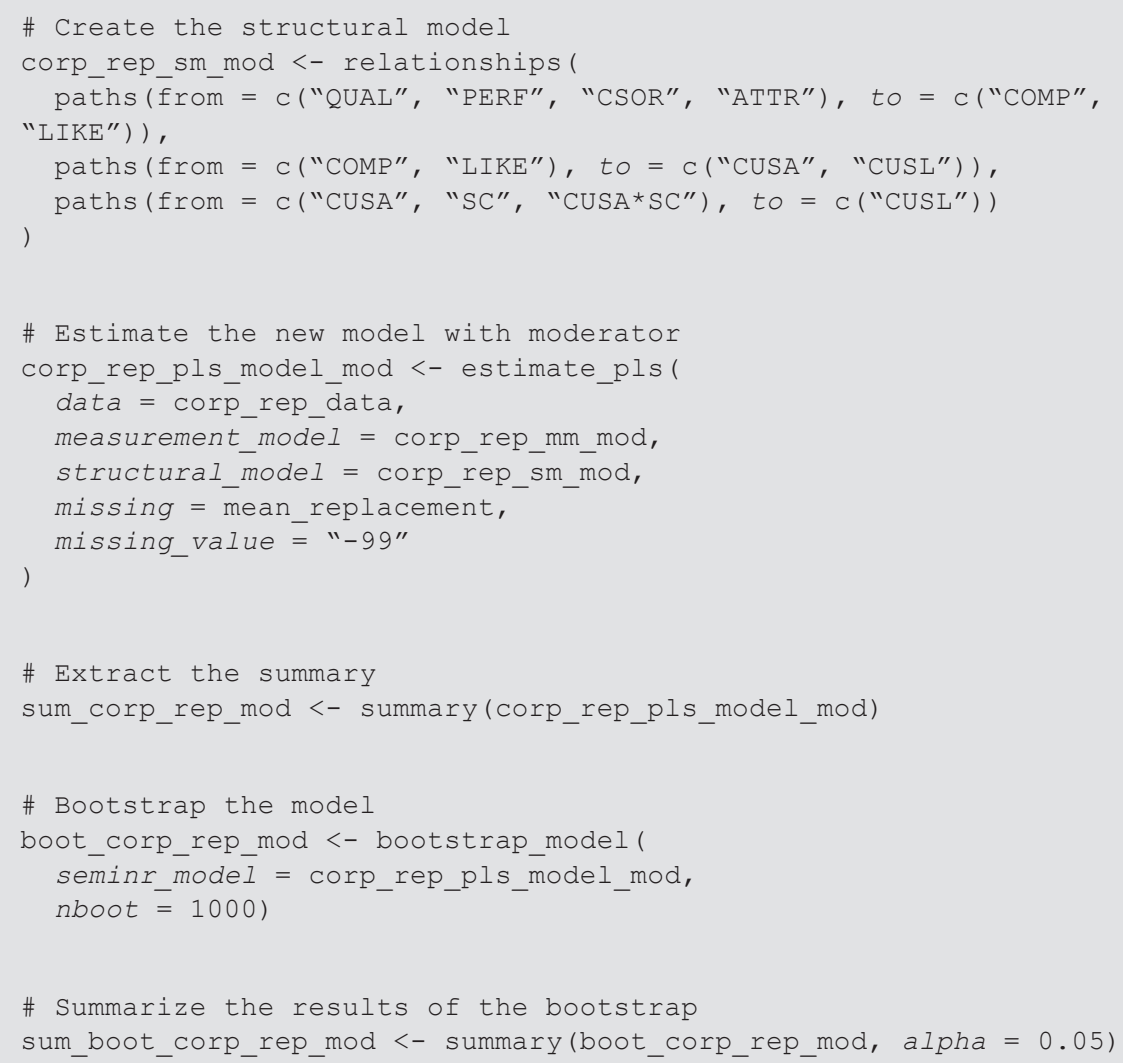

Following the procedures outlined in $>$ Chaps. 4 and 5, we find that all measurement models exhibit sufficient levels of reliability and validity. This also holds for the measures of the newly added SC construct, which exhibit high degrees of internal consistency reliability and convergent validity. In terms of discriminant validity, $S C$ exhibits increased HTMT values only with COMP $(0.850)$ and LIKE (0.802), but these values are significantly lower than 0.90 (Hair et al., 2022; Chap. 7).

Our next concern is the size of the moderating effect. In order to evaluate the moderating effect, we need to inspect the bootstrapped_paths element within the sum_boot_corp_rep_mod object, sum_boot_corp_ rep_mod\$bootstrapped_paths.

\# Inspect the bootstrapped structural paths

sum_boot_corp_rep_mod\$bootstrapped_paths 


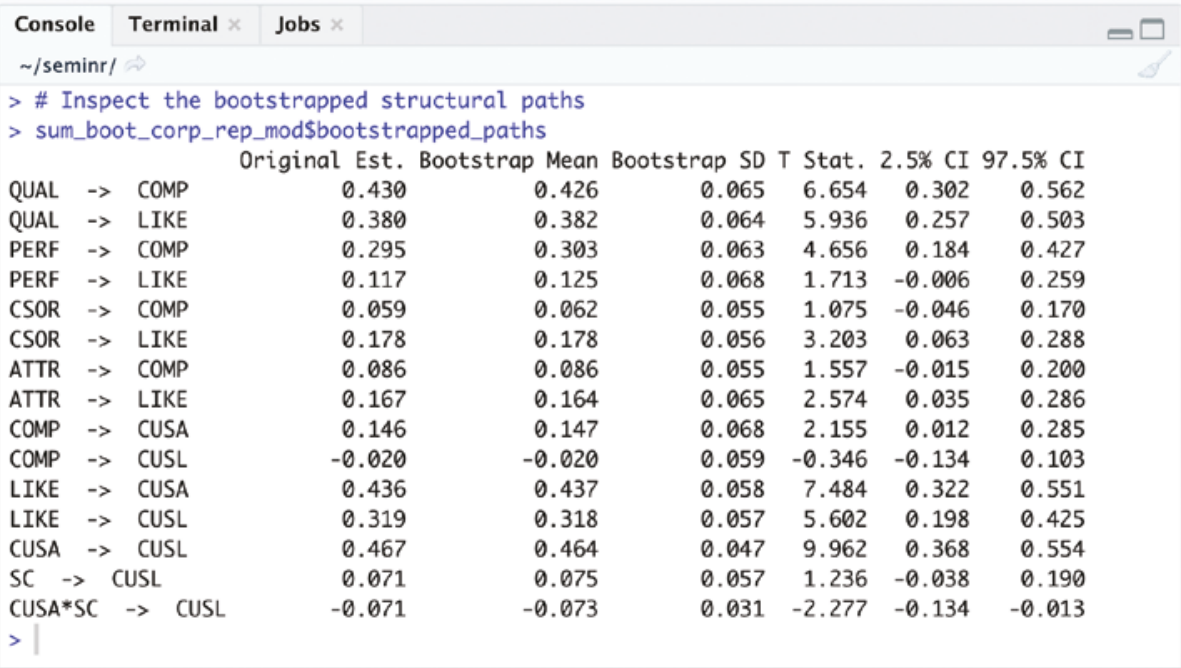

Fig. 8.7 The bootstrapped paths for moderated model. (Source: author's screenshot from R)

As can be seen in - Fig. 8.7, the interaction term $\left(C U S A^{*} S C\right)$ has a negative effect on $C U S L$ of $\mathbf{- 0 . 0 7 1}$, whereas the simple effect of $C U S A$ on $C U S L$ is $\mathbf{0 . 4 6 7}$. Jointly, these results suggest that the relationship between $C U S A$ and $C U S L$ is $\mathbf{0 . 4 6 7}$ for an average level of switching costs. For higher levels of switching costs (i.e., for every standard deviation unit increase of $S C$ ), the relationship between CUSA and $C U S L$ decreases by the size of the interaction term (i.e., $0.467-0.071=0.396$ ). On the contrary, for lower levels of switching costs (i.e., for every standard deviation unit decrease of $S C$ ), the relationship between $C U S A$ and $C U S L$ increases by the size of the interaction term (i.e., $0.467-(-0.071)=0.538)$. To better comprehend the results of the moderator analysis, we can use the slope_analysis () function to visualize the two-way interaction effect (see - Fig. 8.8). This function takes the arguments shown in - Table 8.2.

We apply the slope_analysis() function to the corp_rep_pls_ model_mod, with CUSL as the endogenous construct, $S C$ as the moderator construct, and $C U S A$ as the exogenous construct. Finally, we assign the legend to the bottom-right corner of the plot.

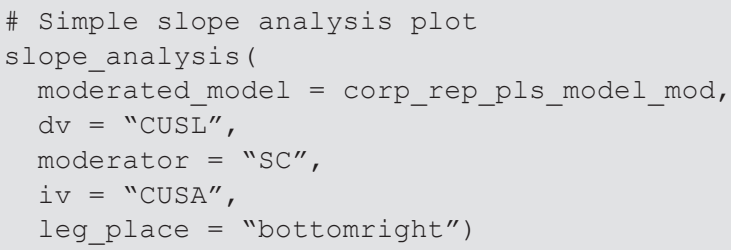

The three lines shown in - Fig. 8.8 represent the relationship between CUSA ( $x$-axis) and CUSL ( $y$-axis). The middle line represents the relationship for an aver- 


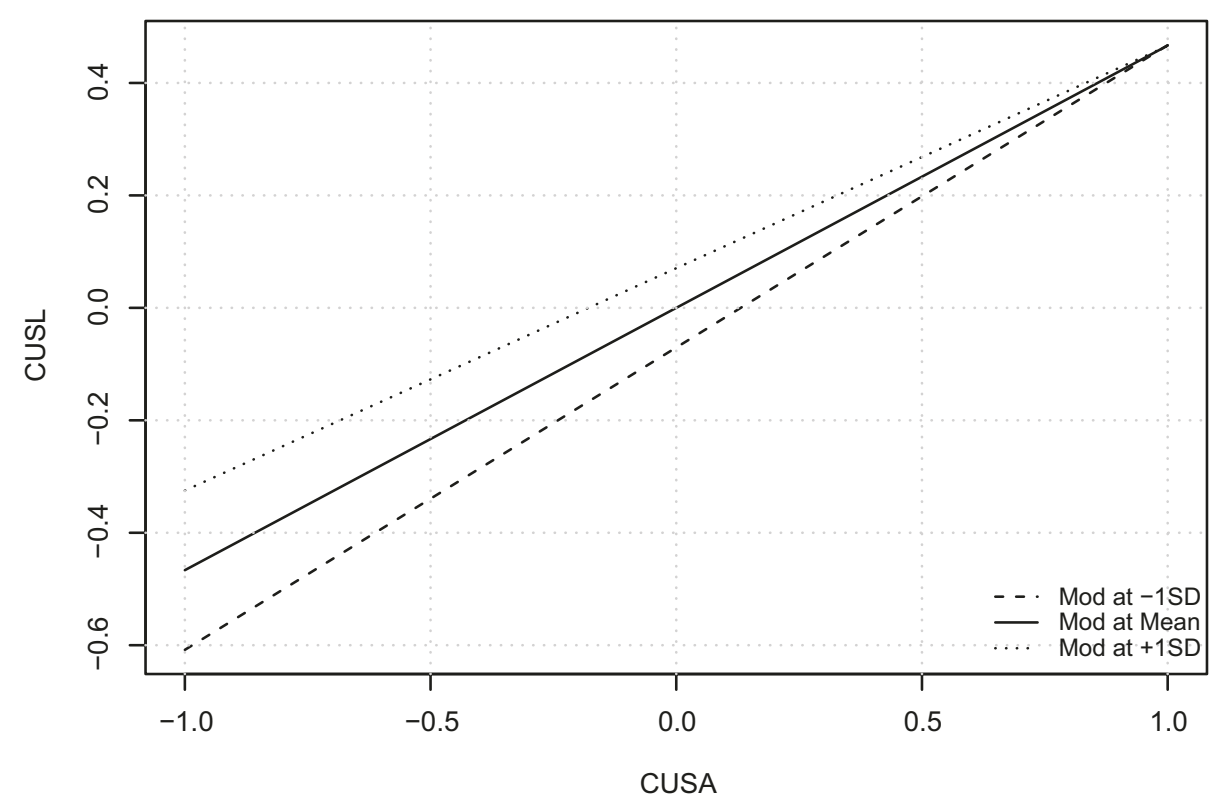

- Fig. 8.8 Simple slope analysis of the two-way interaction effect CUSA*SC on CUSL. (Source: author's screenshot from RStudio)

- Table 8.2 Arguments for the slope_analysis() function

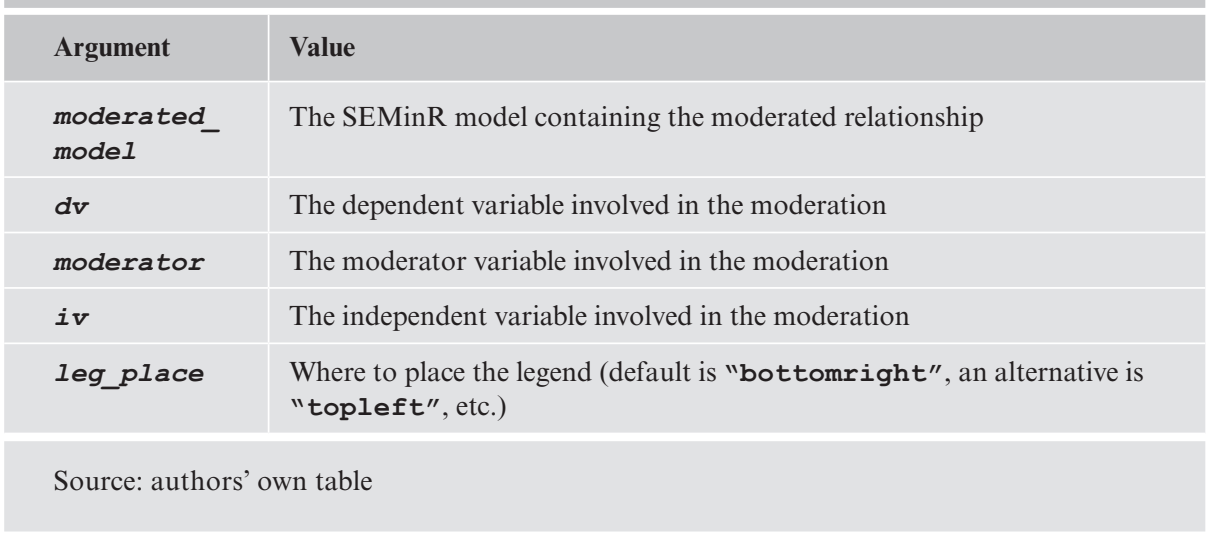

age level of switching costs. The other two lines represent the relationship between CUSA and CUSL for higher (i.e., mean value of $S C$ plus one standard deviation unit) and lower (i.e., mean value of $S C$ minus one standard deviation unit) levels of the moderator variable $S C$. As can be seen, the relationship between CUSA and $C U S L$ is positive for all three lines as indicated by their positive slope. Hence, higher levels of customer satisfaction go hand in hand with higher levels of customer loyalty. Due to the negative moderating effect, at high levels of the modera- 
tor $S C$, the effect of CUSA on CUSL is weaker, while at lower levels of moderator $S C$, the effect of CUSA on CUSL is stronger.

Next, we assess whether the interaction term is significant using the output shown in - Fig. 8.7. The analysis yields a $t$-value of -2.277 for the path linking the interaction term and CUSL. Similarly, the $95 \%$ bootstrap confidence interval of the interaction term's effect is $[-0.134,-0.013]$. As the confidence interval does not include zero, we conclude that the effect is significant. Again, note that these results will slightly differ from yours due to the random nature of the bootstrapping process.

Overall, these results provide clear support that $S C$ exerts a significant and negative moderating effect on the relationship between CUSA and CUSL. The higher the switching costs, the weaker the relationship between customer satisfaction and customer loyalty.

\section{Summary}

Moderation occurs when one construct affects the strength or even the direction of a relationship between two other constructs. As such, moderation accounts for heterogeneity in the data. After theoretically establishing a moderation model, its hypothesized relationships, and the interaction term (generated using the two-stage approach), the model evaluation follows. The moderator construct must be assessed for reliability and validity following the standard evaluation procedures for reflective and formative measurement models. However, this does not hold for the interaction term, which relies on an auxiliary measurement model to represent the interplay between exogenous construct and moderator and their joint effect on the endogenous construct. The result assessment further considers the significance and effect size of the interaction effect. In the simplest form, the analysis considers only one moderator construct, but the model also can involve multiple moderator constructs that can be analyzed simultaneously.

\section{? Exercise}

We continue analyzing the influencer model as introduced in the exercise section of - Chap. 3. Extending the original model ( Fig. 3.10), we hypothesize that perceived influencer competence $(P I C)$ moderates the relationship between perceived quality $(P Q)$ and purchase intention $(P I)$. $P I C$ is measured with reflective items covering traits of the influencer - see Table 3.10 for an overview of the indicators.

- Fig. 8.9 visualizes the model with the $P I C$ and the interaction term $\left(P Q^{*} P I C\right)$ included.

1. Do the measurement models and structural model meet all quality standards?

2. Describe the moderating effect in terms of direction and significance. Visualize the effects using a simple slope analysis.

3. Quantify the moderating effect's size using the $f^{2}$ effect size.

4. What conclusions can you draw from the moderator analysis? 


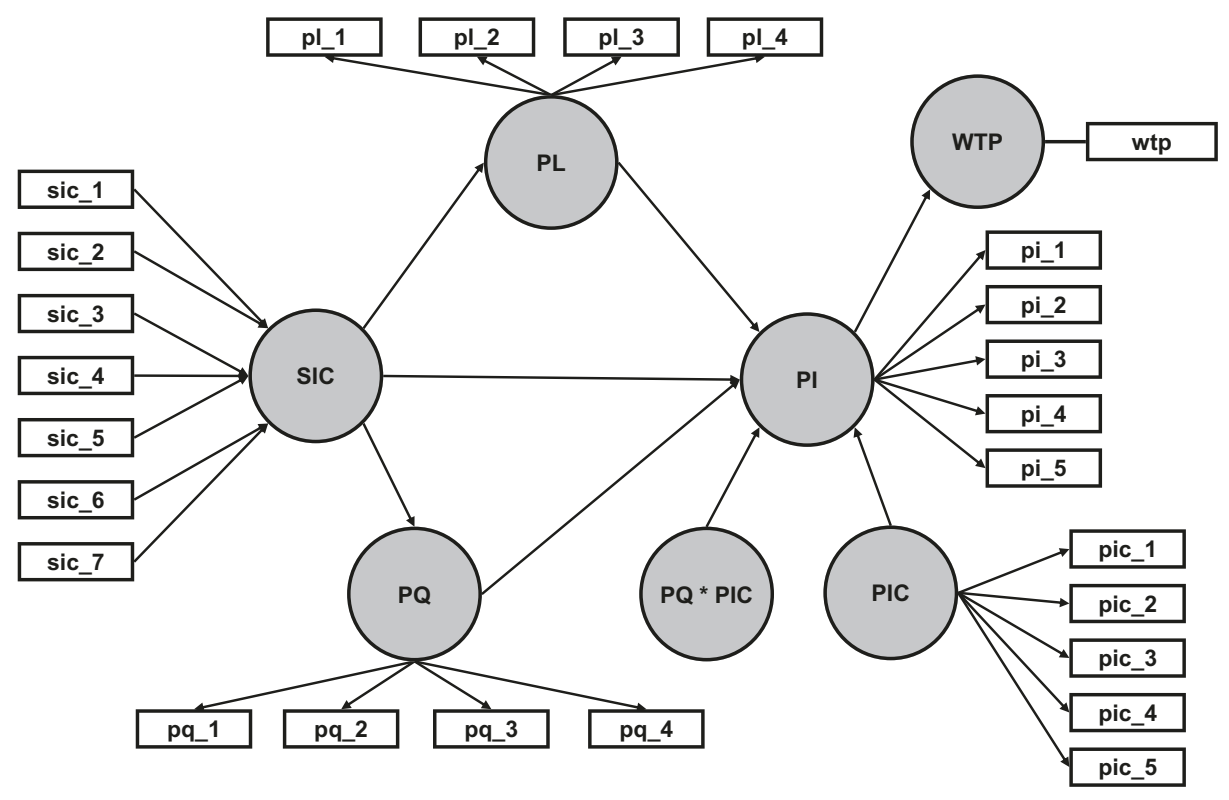

Fig. 8.9 The influencer model with additional hypothesized moderation effect of PIC on PQ (source: authors' own figure)

\section{References}

Aguinis, H., Beaty, J. C., Boik, R. J., \& Pierce, C. A. (2005). Effect size and power in assessing moderating effects of categorical variables using multiple regression: A 30-year review. Journal of Applied Psychology, 90(1), 94-107.

Becker, J.-M., Ringle, C. M., \& Sarstedt, M. (2018). Estimating moderating effects in PLS-SEM and PLSc-SEM: Interaction term generation*data treatment. Journal of Applied Structural Equation Modeling, 2(2), 1-21.

Chin, W. W., Marcolin, B. L., \& Newsted, P. R. (2003). A partial least squares latent variable modeling approach for measuring interaction effects: Results from a Monte Carlo simulation study and an electronic-mail emotion/adoption study. Information Systems Research, 14(2), 189-217.

Cohen, J. (1988). Statistical power analysis for the behavioral sciences. Mahwah, NJ: Lawrence Erlbaum.

Diamantopoulos, A., Sarstedt, M., Fuchs, C., Kaiser, S., \& Wilczynski, P. (2012). Guidelines for choosing between multi-item and single-item scales for construct measurement: A predictive validity perspective. Journal of the Academy of Marketing Science, 40(3), 434-449.

Gardner, R. G., Harris, T. B., Li, N., Kirkman, B. L., \& Mathieu, J. E. (2017). Understanding "it depends" in organizational research: A theory-based taxonomy, review, and future research agenda concerning interactive and quadratic relationships. Organizational Research Methods, 20(4), 610-638.

Hair, J. F., Hult, G. T. M., Ringle, C. M., \& Sarstedt, M. (2022). A primer on partial least squares structural equation modeling (PLS-SEM) (3rd ed.). Thousand Oaks, CA: Sage.

Hair, J. F., Sarstedt, M., Ringle, C. M., \& Gudergan, S. P. (2018). Advanced issues in partial least squares structural equation modeling (PLS-SEM). Thousand Oaks, CA: Sage.

Henseler, J., \& Chin, W. W. (2010). A comparison of approaches for the analysis of interaction effects between latent variables using partial least squares path modeling. Structural Equation Modeling, $17(1), 82-109$. 
Henseler, J., \& Fassott, G. (2010). Testing moderating effects in PLS path models: An illustration of available procedures. In V. Esposito Vinzi, W. W. Chin, J. Henseler, \& H. Wang (Eds.), Handbook of partial least squares: Concepts, methods and applications in marketing and related fields. (Springer Handbooks of Computational Statistics Series, (Vol. II, pp. 713-735). Berlin: Springer.

Homburg, C., \& Giering, A. (2001). Personal characteristics as moderators of the relationship between customer satisfaction and loyalty-An empirical analysis. Psychology and Marketing, 18(1), 43-66.

Jones, M. A., Mothersbaugh, D. L., \& Beatty, S. E. (2000). Switching barriers and repurchase intentions in services. Journal of Retailing, 76(2), 259-274.

Kenny, D. A. (2018). Moderation. Retrieved from http://davidakenny.net/cm/moderation.htm

Klesel, M., Schuberth, F., Niehaves, B., \& Henseler, J. (2021). Multigroup analysis in information systems research using PLS-PM: A systematic investigation of approaches. The DATA BASE for Advances in Information Systems, forthcoming.

Memon, M. A., Cheah, J.-H., Ramayah, T., Ting, H., Chuah, F., \& Cham, T. H. (2018). Moderation analysis: Issues and guidelines. Journal of Applied Structural Equation Modeling, 3(1), i-ix.

Rigdon, E. E., Ringle, C. M., \& Sarstedt, M. (2010). Structural modeling of heterogeneous data with partial least squares. In N. K. Malhotra (Ed.), Review of Marketing Research (pp. 255-296). Armonk, NY: Sharpe.

Sarstedt, M., Diamantopoulos, A., Salzberger, T., \& Baumgartner, P. (2016). Selecting single items to measure doubly-concrete constructs: A cautionary tale. Journal of Business Research, 69(8), 3159-3167.

Sarstedt, M., \& Mooi, E. A. (2019). A concise guide to market research: The process, data, and methods using IBM SPSS statistics (3rd ed.). Berlin: Springer.

\section{Suggested Reading}

Aguinis, H., Edwards, J. R., \& Bradley, K. J. (2016). Improving our understanding of moderation and mediation in strategic management research. Organizational Research Methods, 20(4), 665-685.

Dawson, J. F. (2014). Moderation in management research: What, why, when, and how. Journal of Business and Psychology, 29(1), 1-19.

Fassott, G., Henseler, J., \& Coelho, P. S. (2016). Testing moderating effects in PLS path models with composite variables. Industrial Management \& Data Systems, 116(9), 1887-1900.

Hair, J. F., Hult, G. T. M., Ringle, C. M., \& Sarstedt, M. (2022). A primer on partial least squares structural equation modeling (PLS-SEM) (3rd ed.). Thousand Oaks, CA: Sage.

Memon, M. A., Cheah, J.-H., Ramayah, T., Ting, H., Chuah, F., \& Cham, T. H. (2018). Moderation analysis: Issues and guidelines. Journal of Applied Structural Equation Modeling, 3(1), i-ix.

Open Access This chapter is licensed under the terms of the Creative Commons Attribution 4.0 International License (http://creativecommons.org/licenses/by/4.0/), which permits use, sharing, adaptation, distribution and reproduction in any medium or format, as long as you give appropriate credit to the original author(s) and the source, provide a link to the Creative Commons license and indicate if changes were made.

The images or other third party material in this chapter are included in the chapter's Creative Commons license, unless indicated otherwise in a credit line to the material. If material is not included in the chapter's Creative Commons license and your intended use is not permitted by statutory regulation or exceeds the permitted use, you will need to obtain permission directly from the copyright holder.

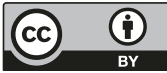

